\title{
ARTICLE
}

\section{What can we learn from offspring studies in bipolar disorder?}

\author{
Josephine Loftus, Bruno Etain \& Jan Scott
}

Josephine Loftus completed her psychiatric training in Ireland and the UK, where she participated in a multicentre genetic linkage project in families with schizophrenia. Her main research interest at that time was the development of clinical phenotypes for genetic studies. She is currently Assistant Clinical Director in an in-patient mood disorder clinic in the Princess Grace Hospital, Monaco and a member of the French Network of Academic Centres of Expertise in Bipolar Disorder. She is about to commence a study of the offspring of parents with bipolar disorders. Bruno Etain completed his

psychiatric training at University Paris V. He is now a Professor of Psychiatry at the University Paris East, and coordinates the French Network of Academic Centres of Expertise in Bipolar Disorders with Professor Chantal Henry. His main research interests include the study of genetic and environmental risk factors for bipolar disorders, with a focus on childhood trauma and chronobiology. Jan Scott is

Professor of Psychological Medicine at the University of Newcastle, and a visiting professor at the Institute of Psychiatry, Psychology and

Neuroscience in London, University of Sydney, and Norwegian University of Science and Technology (NTNU) in Trondheim. She is also a

Distinguished Founding Fellow of the Academy of Cognitive Therapy. Her recent research includes prospectiv studies of risk of bipolar disorders and clinical staging models; and the design and piloting of preventative interventions for young people at risk of bipolar disorders, especially the offspring of parents with bipolar disorders.

Correspondence Dr Josephine Loftus, Princess Grace Hospital, Avenue Pasteur, Monaco 98000 MC Email: jbloftus97@gmail.com

\begin{abstract}
SUMMARY
We offer a contemporary review of studies of the offspring of parents with bipolar disorder and explore the clinical characteristics of these populations. We discuss how different methodological approaches may influence study findings and may explain some of the heterogeneity in the results reported. We also highlight some of the environmental risk factors that may increase the likelihood of transition from an 'at-risk' or high-risk state to bipolar disorder. Last, we briefly discuss the implications of study findings for early intervention strategies and comment on such issues as genetic counselling and primary and early secondary prevention programmes.
\end{abstract}

\section{LEARNING OBJECTIVES}

- Increased knowledge of the methodological variability and findings of studies of the offspring of parents with bipolar disorder

- Increased awareness about illness trajectories and the prevalence of non-mood disorders in the offspring of parents with bipolar disorder

- Understanding of the value of increased vigilance in this population and of any potential role for early intervention strategies

\section{DECLARATION OF INTEREST}

None

Bipolar disorder is a major mental disorder characterised by poor psychosocial outcomes, high medical and psychiatric comorbidity and elevated mortality rates compared with both the general population and most other severe mental disorders. It is the sixth cause of disability-adjusted life years among all diseases in working-age adults (Murray 1996) and is ranked fourth among the global burden of diseases in individuals less than 25 years of age (Gore 2011). Bipolar disorder is also a highly heritable disorder, with up to $85 \%$ of the variance in risk determined by genetic factors, and a positive family history remains the strongest predictive factor for development of the illness. Meta-analyses have shown that, compared with other children, the offspring of a parent with bipolar disorder have an eight- to tenfold increase in their lifetime risk for developing the disorder, a threefold lifetime risk for a major psychiatric disorder and a one-in-two risk for any mental illness (Lapalme 1997; Del Bello 2001; Rasic 2014). Thus, it can be argued that offspring studies provide one of the most reliable and valid means of identifying a sample of individuals at high risk for developing a severe mental disorder and can provide information on prodromal signs and symptoms and rates of transition from an 'at-risk' state to clinical 'caseness'.

In this article, we review how studies of the offspring of parents with bipolar disorder (familialrisk offspring) have enhanced our understanding of the evolution of this illness. We note that longitudinal follow-up of both affected and unaffected high-risk siblings from an early age may reveal clinical, biological and/or environmental factors that predict which high-risk family members ultimately develop bipolar disorder. Prospective studies can potentially clarify the 'illness trajectory' by exposing the temporal relationship between any childhood difficulties or non-mood problems and later affective pathology and/or development of a bipolar disorder. Questions as to whether nonmood pathology is a risk syndrome or earlier manifestation of the disorder, or whether it is a concurrent disorder, may be answered by these studies of high-risk children.

However, studies of high-risk individuals employ many different methodologies, which can complicate cross-study comparisons. Differences include the sampling or recruitment strategy employed (clinical/community; offspring of one affected parent/two affected parents), the type of assessment (self/observer rated) and the time frame for assessments (cross-sectional/longitudinal; retrospective/prospective). To raise awareness of this issue, we begin our overview of offspring studies in bipolar disorder by describing some of the different research approaches used in key studies.

\section{Methodological issues}

\section{Comorbid psychiatric disorders}

Studies published over the past 20 years have reported comparable rates of lifetime DSM Axis 1 disorders and similar increases in the prevalence of affective and non-affective psychopathology 
in the offspring of parents with bipolar disorder compared with offspring of parents without it (controls). However, areas of divergence include the spectrum and prevalence of the psychopathologies observed, the rates and types of comorbidity and the estimated ages at onset of bipolar or other disorders. Duffy et al (2011) have written an important review that highlights why 'methodology matters' in offspring studies in bipolar disorder, and below we also discuss some of the sources of variance in recruitment and assessment strategies that need to be considered when interpreting the published findings.

\section{Recruitment methods and control groups}

The methods employed to recruit families into offspring studies vary from the inclusion of families already involved in neurobiological and genetic research projects (Carlsson 1993; Nurnberger 2011; Egeland 2012), recruitment of all or some of the participants by self-referral and/or via publicity campaigns (Chang 2000; Birmaher 2009, 2010), recruitment from hospital settings and specialised clinics (Klein 1985; Grigoriou-Serbanescu 1989; Hammen 1990; Wals 2001; Henin 2005; Singh 2007) and/or from patient advocacy associations (Wals 2001). It is noteworthy that higher rates of comorbidity were evident in the studies that recruited via selfreferral than in those including bilineal families and families where the non-proband parent also had a non-affective psychiatric illness (see Box 1 for a synopsis of terminology).

Not only does the strategy for recruiting parents and/or offspring vary, but studies differ in the inclusion of any control groups. The mean age of

BOX 1 Some terminology commonly used in offspring studies

Proband The affected index case, i.e. the person serving as the starting point for the genetic study of a family

Non-proband parent Enters the study through their relationship with the proband; they may be an affected or well participant, depending on the study design, but not the initial contact for entering into the genetic study of a family

Unilineal families The disorder is present in one side of the family only: either the maternal or the paternal side, but not both

Bilineal families The disorder is present in both the maternal and the paternal sides of the family

Assortative mating Mating of individuals who have more traits in common than is likely in random mating offspring groups included in studies also varies widely (from 3 to 17 years), rendering direct interview assessment of offspring difficult in the youngest samples and placing excessive reliance on retrospective assessment of any early childhood psychopathology in the older recruits. Likewise, the nature of the control group also influences findings. For example, offspring studies in bipolar disorder that included children of chronically medically ill parents as controls have shown high levels of psychopathology in the control group as well as in the familial-risk group (e.g. Hammen 1990).

It is important to take this methodological heterogeneity into account, as it does appear to influence the reported findings (in parents and offspring). For example, proband parents identified from clinical settings and neurobiological studies had fewer lifetime comorbid diagnoses than those recruited through publicity campaigns, and their socioeconomic status was closer to that reported in community samples, patient contacts with psychiatric services and natural history studies. Almost all studies had a preponderance of female probands with bipolar disorder (the exceptions are: Klein 1985; Egeland 2012), with the proportion varying from $60 \%$ to $100 \%$ (Hammen 1990). A possible explanation of this finding is that there is a 'response bias', i.e. that females with bipolar disorder may be more likely to respond positively to requests to participate in research than their male counterparts. Studies often recruited parents with bipolar I and bipolar II disorders, not taking into account the heterogeneity of bipolar disorder. This may be important, as the clinical presentation, longitudinal course and family history have been shown to differ between different forms of bipolar disorder in adults and failure to take these differences into account could explain discrepant findings (Del Bello 2001; Alda 2004).

\section{Diagnostic assessment and rating tools}

Diagnostic assessments also vary between studies. A best-estimate diagnostic procedure is often used in studies recruiting families who are already involved in neurobiological research, which has the advantage that it can partly overcome the problem of diagnostic instability over time. This approach entails longitudinal and crosssectional assessments as well as an exploration of any treatment history, a review of clinical notes and collection of collateral information from all available and appropriate informants. In contrast, diagnosis in solely cross-sectional studies relies heavily on assessments that evaluate the presence or absence of psychopathology on 
the basis of standardised structured interviews, often conducted by research assistants (Chang 2000; Birmaher 2009, 2010). Some studies employ non-standardised or semi-structured interviews, although these are often carried out by experienced clinicians in clinical rather than community samples (Klein 1985; GrigoriouSerbanescu 1989; Hammen 1990). Dimensional approaches using quantitative rating scales have also been used, although less frequently (Diler 2011; Maoz 2014). The rationale for this is that dimensional assessments may be more sensitive than categorical approaches in detecting the behavioural correlates of early pre-syndromal presentations in the children of parents with bipolar disorder.

A significant proportion of studies used ratings based on parental reports and/or teacher reports and not on direct observation or evaluations of offspring behaviour (Birmaher 2010; Diler 2011; Egeland 2012; Maoz 2014). This may be revealing, as parent ratings may be influenced by their mental state. For example, Birmaher et al (2010) noted that parents reported more distress in their children than did caregivers and teachers. This raises the possibility that ill parents were more sensitive to even transient behavioural difficulties in their children. However, discordant ratings between school and home settings should usually signal the need for further enquiry, as it cannot be assumed that the teachers are always able to give an accurate assessment.

\section{Masking}

Studies also differ in the degree of 'masking' ('blinding') of the researchers undertaking assessments and/or in their access to the children as well as to adult informants. Interviewers of offspring were not always masked to parental diagnosis (Chang 2000; Wals 2001; Egeland 2012). Offspring were not always interviewed directly for cultural (Egeland 2012) or age-related reasons.

\section{The non-proband parents}

A major source of difference between studies is the mental health status of the non-proband parent (Duffy 2011). Given the potential confounding introduced by this 'assortative mating', several researchers have chosen to recruit only families with healthy non-proband parents to circumvent the problem and the additional putative impact of high familial illness burden on the mental health of offspring (Duffy 2010; Egeland 2012). In the studies that do not employ this strategy, rates of DSM Axis 1 disorders in the co-parent vary from $25 \%$ to $49 \%$ (including cases of bipolar spectrum and major depressive disorders) and comorbidities for other disorders may be up to 30\% for substance use disorder and 5\% for disruptive behaviour disorder. Increased rates of psychopathology in the non-proband parent (Chang 2000; Singh 2007; Birmaher 2009, 2010) could also contribute to the prevalence and nature of non-affective psychopathology reported in offspring studies in bipolar disorder. The increased rates of anxiety disorders and disruptive behaviour disorder observed in offspring may be the consequence of genetic factors, increased environmental stressors, or the interaction of environmental and genetic factors through epigenetic mechanisms such as childhood trauma (Etain 2010).

It is also suggested that the inclusion of nonproband parents with psychopathology increases the risk of finding significant associations of pathology such as attention-deficit hyperactivity disorder (ADHD) with bipolar disorder. This point is illustrated in the study by Birmaher et al (2009) in which the prevalence of ADHD and disruptive behaviour disorder was non-significant in the analyses controlled for non-affective psychopathology in the non-proband parent. Several studies have reported high comorbidity for ADHD and bipolar disorder, with rates varying from $11 \%$ to $98 \%$ in children and adolescents in clinical settings (Skirrow 2012). There are several explanations for this wide variation in rates, ranging from overlapping dimensions of psychopathology (e.g. hyperactivity, impulsivity), which lead to over-inflation of comorbidity rates between the two disorders, to the possibility that ADHD-like symptoms may represent a prodromal form of bipolar disorder (Skirrow 2012; Duffy 2014). There is also the question of the diagnosis of bipolar disorder, and mania in particular, in children. In children, manic symptoms, as opposed to manic episodes, transcend diagnostic boundaries (Carlsson 2012). Broadening these boundaries leads to blurring of the distinctions between bipolar disorder and childhood pathologies (such as ADHD) and severe mood dysregulation with depression. These observations underline the importance of longitudinal studies as, for example, these have now shown that a putative 'forme fruste' ' of bipolar disorder, namely severe mood dysregulation syndrome, does not predict later bipolar disorder.

\section{Summary}

This overview has highlighted the dilemmas faced by researchers undertaking offspring studies in bipolar disorder. On the one hand, there is a need to design studies that eliminate possible confounding 
factors, and on the other hand, there is a need to recruit participants who are representative of the clinical and social heterogeneity encountered in these populations. The interpretation of findings from offspring studies should always be accompanied by consideration of the methodology employed (Box 2). Relatively few reviews of the findings of offspring studies (an exception is Duffy 2011) take into account the heterogeneity of bipolar disorder in the parent sample, the impact of co-parent psychopathology, the nature of any control groups, and/or the impact of concomitant life events and/or the family situation. As these are all likely to influence some of the findings, we encourage readers to consider these issues in the future.

\section{What are the findings?}

Having highlighted how methodologies may influence findings, we now review the outcome data from key offspring studies in bipolar disorder (see online Tables DS1 and DS2 in the data supplement for a full list and study details).

\section{Cross-sectional studies}

Most of the key cross-sectional studies listed in Table DS1 found an increased prevalence of major depressive disorder, dysthymia, anxiety disorders and bipolar disorders (bipolar I, bipolar II and bipolar disorder not otherwise specified) in the familial-risk groups. The results include data from three prospective studies that have reported findings from an initial baseline crosssectional assessment of different offspring samples (Birmaher 2009, 2010; Nurnberger 2011).

\section{Bipolar spectrum disorders}

Rates of bipolar spectrum disorders in the familial-risk groups varied from about $2 \%$ to $13 \%$, with lifetime prevalence rates for major affective disorder of $24 \%$. The lowest rate for bipolar spectrum disorder (2\%) was observed in a study of preschool offspring aged 2-5 years (Birmaher 2010). However, this study reported elevated rates for depressive and manic symptomatology in the familial-risk offspring compared with the controls, with differences in manic symptoms remaining significant after correcting for multiple testing. International differences in definitions and recognition of juvenile or paediatric bipolar disorders makes comparisons of early childhood bipolar syndromes difficult (Douglas 2014). For example, prepubertal onset of bipolar disorder in offspring was reported in 12\% in Europe (Henin 2005), but up to $75 \%$ in the USA (Birmaher 2009).
BOX 2 Methodology in offspring studies in bipolar disorder: key points to consider
- Ascertainment and recruitment methods influence findings

- Cross-sectional assessments do not allow for diagnostic instability over time

- Assessment and diagnosis have not always been carried out masked (blind) to family affiliation

- Inclusion of non-proband parents with mental disorders adds potential confounding effects associated with assortative mating and the burden of family illness on offspring

- Studies have not always taken into account the clinical heterogeneity of bipolar disorder
- Offspring have not always been directly interviewed

- Different methodological approaches may target different populations with different biological and environmental risk factors

- The inclusion of different subtypes of bipolar disorder in the adult probands (e.g. bipolar I or bipolar II disorder) may influence findings

- Broader definitions of mania in children may lead to blurring of boundaries with other childhood pathologies such as ADHD and overdiagnosis of paediatric bipolar disorder

\section{Behavioural disorders}

Increased rates of disruptive behaviour disorder and ADHD in young familial-risk offspring were also recorded (Chang 2000; Henin 2005; Singh 2007; Birmaher 2009, 2010); ADHD rates averaged about 25\%, although Chang et al (2000) reported a comorbidity rate of about $80 \%$ for ADHD and bipolar disorder in male offspring. Birmaher et al (2010) also found increased comorbidity for ADHD in familial-risk offspring, but this study reported that both biological parents had significantly higher rates of ADHD, disruptive behaviour disorder, substance use disorder and anxiety disorders than the control parents. Birmaher et al's (2009) earlier study did not find any difference in rates of ADHD and disruptive behaviour disorder between familialrisk offspring and offspring of the control parents. Some researchers suggest that the disruptive behaviour disorder and ADHD observed in the familial-risk offspring may be related to general parental psychopathology and not specifically to bipolar disorder. However, the patterns are difficult to interpret in cross-sectional studies. For example, Vandeleur et al (2012) reported an increased risk for disruptive behaviour disorder in the offspring of parents concordant for major depressive disorder but not in the offspring of parents with bipolar disorder.

Findings reported by Birmaher et al (2009) also suggest a possible interaction between socioeconomic status (SES) and disruptive behaviour disorders, with high SES appearing to act as a protective factor against disruptive behaviour disorder in the offspring of high-SES healthy 
controls only (and not in the offspring of lowSES healthy controls, or in the offspring of highor low-SES parents with bipolar disorder). The importance of the social and familial context was also highlighted by Vandeleur et al (2012). They observed that the association between mood disorder in the proband and offspring was weakened when the familial situation, namely 'living with both biological parents', was taken into account. One interpretation is that this is an additional factor which influences the probability of onset of a mood disorder in 'at-risk' offspring.

\section{Anxiety disorders}

Most studies found increased anxiety disorders in offspring of parents with bipolar disorder (Chang 2000; Henin 2005; Birmaher 2009; Vandeleur 2012), supporting the possibility that anxiety may be an early marker for bipolar disorder. Also, three studies (Chang 2000; Birmaher 2009; Vandeleur 2012) reported increased rates of depressive symptoms in offspring of bilineal families as opposed to children from unilineal families (Box 1). However, a study of families with only bipolar I disorder (Singh 2007) did not report this finding.

\section{Dimensional psychopathy}

Several research groups over the past decade have included validated dimensional scales in their assessments (Dienes 2002). These scales have included the Child Behavior Checklist (CBCL), the Emotionality Activity Sociability (EAS) survey and the Early Child Inventory (ECI) (Diler 2011; Maoz 2014). The studies have generated rather mixed results. For example, Diler et al (2011) reported that school-age offspring of parents with bipolar disorder scored significantly higher than controls on subscales measuring anxious, depressed and aggressive behaviours. Although these results were interpreted as meaning that these features may be potential early markers for bipolar disorder, it should be noted that the highest scores were recorded in those offspring who already met diagnostic criteria for a bipolar disorder, whereas unaffected offspring had scores that were intermediate between the affected offspring and the control group. A problem with this (and the other dimensional studies), however, is that high scores on the CBCL, the EAS and the ECI are reported in studies of $\mathrm{ADHD}$, anxiety and post-traumatic stress disorder, and so are probably non-specific to bipolar disorder (Ayer 2009; Meyer 2009).

A study of preschool children in Pittsburgh, USA (Maoz 2014) also found higher levels of dimensional psychopathology in the offspring of parents with bipolar disorder compared with the offspring of unaffected parents, with the former demonstrating higher global scores on the CBCL and on its externalising, somatic, sleep and aggression subscales. Interestingly, scores on the CBCL, the ECI (sleep and anxiety subscales) and the EAS (emotionality) were significantly elevated in the offspring of parents with bipolar II disorder by comparison with the offspring of parents with bipolar I disorder. The differences remained significant after controlling for demographic and parental clinical variables, which suggests that higher levels of dimensional psychopathology were not due to familial stressors or a higher prevalence of disorders such as ADHD. The higher levels of anxiety measured by the CBCL and ECI in these preschool children were non-significant after controlling for other factors, which suggests that anxiety may be associated with general familial stressors and not specifically with bipolar disorder. In contrast, sleep disturbances, aggression, mood instability and somatic complaints remained significant.

Last, Diler et al (2011) did not observe any association between perceived attachment and risk for psychopathology in a school-age group of offspring of parents with bipolar disorder.

\section{Prospective longitudinal studies}

In one of the first published offspring studies in bipolar disorder, Akiskal et al (1985) reported increased rates of anxiety disorders, minor mood disorders and adjustment disorders in a small sample $(n=68)$ of children and younger siblings of adults with bipolar disorder investigated over a 3-year period (Table DS2). Affective disorders began in adolescence and were depressive in nature (median age at onset $\sim 16$ years). Subsequent longitudinal studies with larger samples and longer follow-up periods of 12,15 and 16 years have largely replicated those findings (Duffy 2010; Egeland 2012; Mesman 2013).

All of the prospective studies shown in Table DS2 reported increased mood and non-mood psychopathology in the offspring of parents with bipolar disorder compared with offspring of healthy controls. However, in contrast to retrospective reporting of childhood-onset bipolar disorder in the cross-sectional studies (Table DS1), none of the prospective studies has reported prepubertal onset of mania. The prospective studies consistently report that the first major affective episode in familial-risk offspring is depressive in polarity and usually begins in mid-to-late adolescence. Also the depressive episode precedes the first (hypo) manic episode by about 3-5 years. Although the trajectories show some consistency (non-specific 
childhood problems or anxiety, followed by depression, then by mania), it is noteworthy that the overall transition rates to syndromal bipolar disorder are low. Having said that, many of the studies were instigated in the past decade, and not all the familial-risk samples have passed through the peak period for onset of bipolar disorder. For example, Egeland et al (2012) calculated that $68 \%$ of their sample (age range: 13-29 years) were still within the period of maximum risk for onset of bipolar I disorder, while the mean ages at follow-up in the studies by Duffy et al (2010) and Mesman et al (2013) were about 24 (range 8-30) and 28 (s.d.=3) years respectively. As more follow-up data become available it is possible that there will be a small increment in 'caseness' in the familial-risk samples.

Three studies recorded detailed illness trajectories for their study cohorts (Duffy 2010; Egeland 2012; Mesman 2013). These showed similar patterns, with the emergence of non-mood psychopathology (such as anxiety symptoms, sleeping problems and increased sensitivity in early childhood), with progression to adjustment disorders, mood symptoms and minor mood disorders, followed by the onset of a major affective episode. Duffy et al (2010) reported that $71 \%$ of cohort participants demonstrated this temporal sequence of psychopathology and that preceding anxiety increased the age-adjusted risk of mood disorder from $40 \%$ to $85 \%$. Also the presence of a mood disorder increased the morbid age-adjusted risk of substance misuse to $35 \%$, compared with $18 \%$ in participants without mood disorders. Mesman et al (2013) observed a later onset of comorbid anxiety in offspring with a bipolar spectrum disorder than Duffy et al (2010), although this may have reflected the later mean age at enrolment. In the Dutch study, prevalence rates for substance use disorder were similar to those found in the general population and may have reflected recruitment methods, as participants were recruited from patient associations who were sensitised to the effects of substance misuse on bipolar disorder (Mesman 2013).

In the USA, Egeland et al (2012) observed progression of symptoms, with early-childhood anxiety, worry and increased sensitivity giving way at mid-adolescence to decreased concentration, high energy, excessive talking, unruly behaviour and depressed mood. Egeland et al did not report increased substance use disorder in their sample, but this might be partly explained by cultural factors, as they recruited families from the Amish community (a religious group who are largely abstinent).
It is important to note that none of the prospective longitudinal offspring studies demonstrates an increased rate of ADHD, various learning difficulties, childhood manic or hypomanic episodes among offspring of parents with bipolar disorder. This is contrary to the findings of crosssectional and community-based studies (Faedda 2014). The absence of an increased rate of ADHD in the Amish study is particularly interesting, given the characteristics of the population investigated (high genetic loading and sociocultural buffers such as strong social links, minimal rates of substance use disorder, etc.). However, it is clear that the associations between ADHD and bipolar disorder are still being investigated. For example, Duffy et al (2014) described a subgroup of offspring of 'lithium non-responder' parents with bipolar disorder, who, in comparison with offspring of 'lithium-responder' parents with the disorder, had double the rates of ADHD, learning disability (intellectual disability) antecedents and DSM Cluster A personality traits and nonremitting mood disorders (14\% v. 8\%). However, the differences were not statistically significant, possibly owing to insufficient sample size. Also, all cases of schizoaffective disorder were observed in the offspring of lithium non-responder parents. Duffy etaldiscussed several explanations, including the possibility that there may be a subtype of bipolar disorder characterised by the emergence of 'ADHD-like' behaviours in childhood and early onset of bipolar disorder associated with psychotic symptoms and resistance to lithium treatment.

\section{Who makes the transition to bipolar disorder and why?}

About $10 \%$ of the offspring of parents with bipolar disorder will themselves develop bipolar disorder. As concordance rates for bipolar disorder never reach $100 \%$ even in monozygotic twins, it is likely that other factors are involved in the transition from 'at-risk' status to 'caseness' (affected status). Genetic and environmental risk factors may interact to precipitate onset and modify the clinical course of bipolar disorder in at-risk individuals.

\section{Paternal and maternal age}

Advanced paternal age has been reported as a risk factor for neurodevelopmental disorders and studies have demonstrated an increased risk for bipolar disorder in offspring of older fathers $(>50)$ in comparison with the offspring of younger fathers (Frans 2008; Chudal 2014). GrigoriouSerbanescu et al (2012) reported paternal age to have the greatest effect on age at onset of sporadic 
(non-familial) bipolar I disorder, especially in females. In contrast, all studies have failed to show a relationship between maternal age and risk for bipolar disorder, with the exception of one by Menezes et al (2010).

\section{Gestational factors}

A study by Wals et al (2003) demonstrated an association between low birth weight and both affective and non-affective disorders in familialrisk offspring that was independent of familial loading for bipolar disorder, unipolar disorder and substance use disorder. Other environmental factors interacting with the underlying genotype may be early parental loss (Mortensen 2003) and maternal influenza during pregnancy (Parboosing 2013). Although there are conflicting results regarding the latter, a recent study suggested that maternal influenza was not associated with an increased risk of bipolar disorder in offspring, but was associated with a fivefold risk of bipolar disorder with psychotic features (Canetta 2014).

\section{Socioenvironmental factors}

Offspring of parents with bipolar disorder are reported to live in more dysfunctional families than children of parents without mental disorders (Chang 2001; Moreno 2012), and the studies of Ferreira et al (2013), Vandeleur et al (2012) and Hammen et al (1990) all reported an association between living in such an environment and the risk of psychopathology in the former group. Also, Hillegers et al (2004) demonstrated that stress load (frequency of life events) increased the liability to develop mood disorders in familial-risk offspring during adolescence. In wider research, it is hypothesised that people who develop bipolar disorder are more likely to have been raised in families that are characterised by less cohesion and organisation, more conflicts (Chang 2001; Romero 2005), less reward (Heru 2004) or with higher levels of expressed emotion, including critical, hostile or overinvolved attitudes (Ogilvie 2005). Burden on the informal carers of people with bipolar disorder is also associated with more depressive symptoms, which could result in lowquality interactions between parents and their affected offspring or even to increased levels of emotional neglect (Ogilvie 2005).

\section{Substance misuse}

Duffy et al (2012) found that substance use disorder in high-risk offspring was associated with a threefold increase in the risk of developing a mood disorder and also with an increased risk of developing psychotic symptoms. The relationship between substance use disorder, in particular cannabis misuse, and bipolar disorder is complex. The strong bidirectional associations may reflect gene-environment interactions involving several different genes and environmental factors, including the increased vulnerability of the adolescent brain to the toxic effects of cannabis (Henquet 2008); it might also be associated with an above average level of risk-taking behaviour in those at risk of bipolar disorders or an attempt at 'self-medication' in those with recentonset bipolar disorder.

\section{Early interventions}

One of the potential problems of offspring studies is that they usually employ healthy controls rather than positive controls (i.e. parents with a different mental disorder, such as psychosis). This may engender a false sense of optimism with regard to the specificity of the illness trajectories for bipolar disorder, when in fact, research in other offspring groups and in youth mental health suggests that heterotypical continuity from non-specific to more specific syndromes (especially anxiety to depression to specific disorder) is a typical trajectory for most severe mental disorders (Berk 2009; Scott 2013). Therefore interventions that target anxiety, sleep or other childhood problems that have been shown to occur more often in offspring of parents with bipolar disorder than in controls may be offering general rather than specific prevention. Nonetheless, recent publications argue that it is timely to consider the options for primary prevention or early secondary prevention of bipolar disorder (Vallarino 2014, 2015). Such approaches might include interventions that target familial-risk offspring who demonstrate nonspecific or subsyndromal psychopathology, but it is interesting to consider whether there are any supportive interventions that should be offered to healthy, asymptomatic familial-risk offspring. We will briefly consider two options, genetic counselling and psychological interventions.

\section{Genetic counselling}

Genetic testing is now commercially available in some countries, and can even be bought over the counter at a national chain store in the UK. Although this is seen as an advance by some, it also raises significant concerns, as there is a lack of regulation and supportive genetic counselling services (Delisi 2014). In bipolar disorders, attitudes to genetic testing have been explored with patients and their families (Jones 2002; Meiser 2005). These studies have shown that the majority of participants expressed an interest in 
testing if it would provide a definite answer. About half of the individuals questioned were in favour of genetic testing for at-risk adolescents, but only if early preventive therapeutic interventions were available.

However, genetic testing is not synonymous with genetic counselling, and the latter may be important regardless of the availability of a test. Some education about the likelihood of inheritance is important for families as, at the very least, misunderstandings such as an overestimation of risk can be clarified (Hill 2006). Genetic counselling can also alert families to the concurrent risk of non-mood disorders within families and encourage better management of risk through increased awareness of environmental stressors or modifiable factors. Anecdotal evidence suggests, however, that parents with bipolar disorder are sometimes keener on input for their offspring than are the young people themselves. Although many do wish to understand the possible risk, others express the view that they prefer to 'live their lives' in the same way as their peer group, and do not wish to consider using additional selfmanagement strategies (such as sleep hygiene), for fear of medicalisation or stigmatisation.

\section{Psychological interventions}

In asymptomatic young people who wish to explore stress management, two promising approaches would appear to be sleep regulation and anti-rumination strategies. The former has greater impact on risk for elevated or unstable mood, and the latter for depressed mood or substance use (Vallarino 2014). In those who manifest other problems or subsyndromal mood symptoms, attenuated models of currently available psychological therapies such as cognitive-behavioural therapy, interpersonal therapy or family-focused therapy can all improve sleep, reduce depression and improve functioning (Vallarino 2014, 2015). The only randomised trial to date in offspring of parents with bipolar disorder demonstrates that family-focused therapy may be especially useful to families with high levels of expressed emotion, but that gains for other families are modest compared with usual treatments (Miklowitz 2014).

\section{Conclusions}

The offspring of parents with bipolar disorder provide an enriched sample for longitudinal studies of the early stages of bipolar disorder and exploration of illness trajectories in high-risk subgroups. Despite methodological differences, nearly all the available studies agree on the higher prevalence of mood and non-mood disorders in these young people and that many develop psychopathology at an earlier age than the offspring of parents without bipolar disorder (Box 3). Studies of disease progression from the asymptomatic or latent phase to development of the disorder appear to support the applicability of a clinical staging approach to bipolar disorder (Scott 2013). Offspring studies confirm the heritability of the disorder, but also emphasise the need to study the environmental factors involved in the transition from high-risk to affected status.

The presence of non-specific psychopathology may offer opportunities for early intervention strategies to avoid development of mania or other severe problems. Offspring studies also raise awareness of the high prevalence of nonmood disorders in this population. Options for interventions are only just being considered, but it can be argued that some form of education about risks needs to be available for these young people, although this should not necessarily extend to genetic counselling. This input would not only increase awareness, but also offer hope for the future and reinforce the fact that the vast majority of children of parents with bipolar disorder (i.e. about 90\%) do not develop the disorder.

BOX 3 Studies of offspring of parents with bipolar disorder: key findings

- Increased rates of bipolar disorder and DSM Axis 1 diagnoses were reported in offspring

- Offspring who developed bipolar disorder often displayed non-specific symptoms in childhood

- A typical trajectory for the development of bipolar disorder, reported in 10-30\% of offspring who reach 'caseness' is: early non-specific symptoms and signs in childhood that progress to minor mood disorders, followed by a major depressive episode in midadolescence, with a first episode of (hypo)mania about 3-5 years later

- Prepubertal mania was rare and when it was reported it tended to be in cross-sectional studies or in retrospective assessments

- ADHD rates were high in cross-sectional studies, but rates were either not increased or not predictive of onset of bipolar disorder in longitudinal studies

- Dimensional studies suggest that somatic complaints sleep disturbances, high emotionality and aggression in the context of a positive family history of bipolar disorder may predict progression to illness status

- The presence of a mood disorder increased the ageadjusted morbid risk of substance use disorder from $18 \%$ (in participants without mood disorder) to $35 \%$ 


\section{MCO answers}

$\begin{array}{lllll}1 a & 2 b & 3 c & 4 b & 5 b\end{array}$

\section{References}

Akiskal H, Downs J, Jordan P, et al (1985) Affective disorders in referred children and younger siblings of manic-depressives: mode of onset and prospective course. Archives of General Psychiatry, 42: 996-1003.

Alda M (2004) The phenotypic spectra of bipolar disorder. European Neuropsychopharmacology, 14 (suppl 2): S94-9.

Ayer L, Althoff R, Ivanova M, et al (2009) Child Behavior Checklist Juvenile Bipolar Disorder (CBL-JBD) and CBCL Posttraumatic Stress Problems (CBCL-PTSD) scales are measures of a single dysregulatory syndrome. Journal of Child Psychology, 50: 1291-300.

Berk M, Malhi GS, Hallam K, et al (2009) Early intervention in bipolar disorders: clinical biochemical and neuroimaging imperatives. Journal of Affective Disorders, 114: 1-13

Birmaher B, Axelson D, Monk K, et al (2009) Lifetime psychiatric disorders in school-aged offspring of parents with bipolar disorder: the Pittsburgh Bipolar Offspring study. Archives of General Psychiatry, 66: 287-96.

Birmaher B, Axelson D, Goldstein B, et al (2010) Psychiatric disorders in preschool offspring of parents with bipolar disorder: the Pittsburgh Bipolar Offspring Study (BIOS). American Journal of Psychiatry, 167: 321-30.

Canetta SE, Bao Y, Ennis FA, et al (2014) Serological documentation of maternal influenza exposure and bipolar disorder in adult offspring. American Journal of Psychiatry, 171: 557-63.

Carlsson GA (2012) Differential diagnosis of bipolar disorder in children and adolescents. World Psychiatry, 11: 146-52.

Carlsson GA, Weintraub S (1993) Childhood behavior problems and bipolar disorder: relationship or coincidence. Journal of Affective Disorders, 28: 143-53.

Chang KD, Steiner H, Ketter TA (2000) Psychiatric phenomenology of child and adolescent bipolar offspring. Journal of the American Academy of Child \& Adolescent Psychiatry, 39: 453-60.

Chang KD, Blasey C, Keller TA, et al (2001) Family environment of children and adolescents with bipolar parents. Bipolar Disorders, 3: 73-8.

Chudal R, Gissler M, Sucksdorff D, et al (2014) Parental age and risk of bipolar disorders. Bipolar Disorders, 16: 624-32.

Del Bello MP, Geller B (2001) Review of studies of child and adolescent offspring of bipolar parents. Bipolar Disorders, 3: 325-34.

Delisi L, Lynn E (2014) Ethical issues in the use of genetic testing of patients with schizophrenia and their families. Current Opinion in Psychiatry, 27: 191-6.

Dienes KA, Chang KD, Blasey CM, et al (2002) Characterization of children of bipolar parents by parent report CBCL. Journal of Psychiatric Research, 36: 337-45.

Diler RS, Birmaher B, Axelson D (2011) Dimensional psychopathology in offspring of parents with bipolar disorder. Bipolar Disorder, 13: 670-8.

Douglas J, Scott J (2014) A systematic review of gender-specific rates of unipolar and bipolar disorders in community studies of pre-pubertal children. Bipolar Disorders, 16: 5-15.

Duffy A, Alda M, Hajek T, et al (2010) Early stages in the development of bipolar disorder. Journal of Affective Disorders, 121: 127-35.

Duffy A, Doucette S, Lewitzka U, et al (2011) Findings from bipolar offspring studies: methodology matters. Early Intervention in Psychiatry, 5: 181-91.

Duffy A, Horrocks J, Milin R, et al (2012) Adolescent substance use disorder during the early stages of bipolar disorder: a prospective highrisk study. Journal of Affective Disorders, 142: 57-64.

Duffy A, Horrocks J, Doucette S, et al (2014) The developmental trajectory of bipolar disorder. British Journal of Psychiatry, 204: 122-8.

Egeland JA, Endicott J, Hostetter AM, et al (2012) A 16-year prospective study of prodromal features prior to BP onset in well Amish children. Journal of Affective Disorders, 142: 186-92.

Etain B, Mathieu F, Henry C, et al (2010) Preferential association between childhood emotional abuse and bipolar disorder. Journal of Trauma Stress, 23: 376-83.
Faedda GL, Serra G, Marangoni C, et al (2014) Clinical risk factors for bipolar disorders: a systematic review of prospective studies. Journal of Affective Disorders, 168: 314-21.

Ferreira GS, Moreira CR, Kleinman A, et al (2013) Dysfunctional family environment in affected versus unaffected offspring of parents with bipolar disorder. Australian and New Zealand Journal of Psychiatry, 47: 1051-7.

Frans EM, Sandin S, Reichenberg A, et al (2008) Advancing paternal age and bipolar disorder. Archives of General Psychiatry, 65: 1034-40.

Gore FM, Bloem PJ, Patton GC, et al (2011) Global burden of disease in young people aged 10-24 years: a systematic analysis. Lancet, 377: 2093-102

Grigoriou-Serbanescu M, Christodorescu D, Jipescu I, et al (1989) Psychopathology in children aged 10-17 of bipolar parents: psychopathology rate and correlates of the severity of the psychopathology. Journal of Affective Disorders, 16: 167-79.

Grigoriou-Serbanescu M, Wickramaratne PJ, Mihailescu R, et al (2012) Paternal age effect on age of onset in bipolar I disorder is mediated by sex and family history. American Journal of Medical Genetics Part B: Neuropsychiatric Genetics, 195B: 567-79.

Hammen C, Burge D, Burney E, et al (1990) Longitudinal study of diagnosis in children of women with unipolar and bipolar affective disorder. Archives of General Psychiatry, 47: 1112-7.

Henin A, Biedermann J, Mick E, et al (2005) Psychopathology in the offspring of parents with bipolar disorder: a controlled study. Biological Psychiatry, 58: 554-61.

Henquet C, Di Forti M, Morrison P, et al (2008) Gene-environment interplay between cannabis and psychosis. Schizophrenia Bulletin, 34: 1111-21.

Heru AM, Ryan CE (2004) Burden, reward and family functioning of caregivers for relatives with mood disorders: 1-year follow-up. Journal of Affective Disorders, 83: 221-5.

Hill MK, Sahhar M (2006) Genetic counselling for psychiatric disorders. Medical Journal of Australia, 185: 507-10.

Hillegers MHJ, Burger H, Wals M, et al (2004) Impact of stressful life events, familial loading and their interaction on the onset of mood disorders: study in a high-risk cohort of adolescent offspring of parents with bipolar disorder. British Journal of Psychiatry, 185: 97-101.

Jones I, Scourfield J, McCandless F, et al (2002) Attitudes towards testing for future bipolar susceptibility genes: a preliminary investigation. Journal of Affective Disorders, 71: 189-93.

Klein DN, Depue RA, Slater JF (1985) Cyclothymia in the adolescent offspring of parents with bipolar affective disorder. Journal of Abnormal Psychology, 94: 115-27.

Lapalme M, Hodgins S, LaRoche C (1997) Children of parents with bipolar disorder: a metaanalysis of risk for mental disorders. Canadian Journal of Psychiatry, 42: 623-31.

Maoz H, Goldstein T, Axelson DA, et al (2014) Dimensional psychopathology in preschool offspring of parents with bipolar disorder. Journal of Child Psychology and Psychiatry, 55: 144-53.

Meiser B, Mitchell PB, McGirr H, et al (2005) Implications of genetic risk information in families with a high density of bipolar disorder: an exploratory study. Social Science \& Medicine, 60: 109-18.

Menezes PR, Lewis G, Rasmussen F, et al (2010) Paternal and maternal ages at conception and risk of bipolar disorder in their offspring. Psychological Medicine, 40: 477-85.

Mesman E, Nolen WA, Reichart CG, et al (2013) The Dutch bipolar offspring study: 12-year follow-up. American Journal of Psychiatry, 170: 542-9.

Meyer SE, Carlson GA, Youngstrom E, et al (2009) Long-term outcomes of youth who manifested the CBCL-Pediatric Bipolar Disorder phenotype during childhood and/or adolescence. Journal of Affective Disorder, 113: 227-35

Miklowitz DJ, Schneck CD, George EL, et al (2014) Pharmacotherapy and family-focused treatment for adolescents with bipolar I and II disorders: a 2-year randomized trial. American Journal of Psychiatry, 171: 658-67. 
Moreno DH, Bio DS, Petresco S, et al (2012) Burden of maternal bipolar disorder on at-risk offspring: a controlled study on family planning and maternal care. Journal of Affective Disorders, 143: 172-8.

Mortensen PB, Pedersen CB, Melbye M, et al (2003) Individual and familial risk factors for bipolar affective disorders in Denmark. Archives of General Psychiatry, 60: 1209-15.

Murray CJL, Lopez AD (eds) (1996) The Global Burden of Disease: A Comprehensive Assessment of Morbidity and Disability from Diseases, Injuries and Risk Factors in 1990 projected to 2020. Harvard University Press.

Nurnberger JL Jr, McInnis M, Reich W, et al (2011) A high-risk study of bipolar disorder: childhood clinical phenotypes as precursors of major mood disorders. Archives of General Psychiatry, 68: 1012-20.

Ogilvie AD, Morant N, Goodwin GM (2005) The burden on informal caregivers of people with bipolar disorder. Bipolar Disorders, 7 (suppl 1): $25-32$

Parboosing R, Bao Y, Shen L, et al (2013) Gestational influenza and bipolar disorder in adult offspring. JAMA Psychiatry, 70: 677-85

Rasic D, Hajek T, Alda M, et al (2014) Risk of mental illness in offspring of parents with schizophrenia, bipolar disorder, and major depressive disorder: a meta-analysis of family high-risk studies. Schizophrenia Bulletin, 40: 28-38.

Romero S, Delbello MP, Soutullo CA, et al (2005) Family environment in families with versus families without parental bipolar disorder: a preliminary comparison study. Bipolar Disorders, 7: 617-22
Scott J, Leboyer M, Hickie I, et al (2013) Clinical staging in psychiatry: a cross-cutting model of diagnosis with heuristic and practical value. British Journal of Psychiatry, 202: 243-5.

Singh MK, DelBello MP, Stanford KE, et al (2007) Psychopathology in children of bipolar parents. Journal of Affective Disorders, 102: 131-6.

Skirrow C, Hosang GM, Farmer AE, et al (2012) An update on the debated association between ADHD and bipolar disorder across the lifespan. Journal of Affective Disorders, 141: 143-59.

Vallarino M, Scott J (2014) State of the art: psychotherapies for individuals at risk, or experiencing a first episode, of bipolar disorder. In Mental Health in Adolescents: Bipolar Disorder (Clinical Insights series) (ed A Duffy): 127-43. Future Medicine.

Vallarino M, Scott J (2015) An evidence map of psychosocial interventions for the earliest stages of bipolar disorders. Lancet Psychiatry, 2: $548-63$

Vandeleur C, Rothen S, Gholam-Rezaee M, et al (2012) Mental disorders in offspring of parents with bipolar and major depressive disorders. Bipolar Disorders, 14: 641-53.

Wals M, Hillegers MH, Reichart CG, et al (2001) Prevalence of psychopathology in children of a bipolar parent. Journal of the American Academy of Child \& Adolescent Psychiatry, 40: 1094-102.

Wals M, Reichart CG, Hillegers MH, et al (2003) Impact of birth weight and genetic liability on psychopathology in children of bipolar parents. Journal of the American Academy of Child \& Adolescent Psychiatry, 42 . 1116-21.

\section{MCOs}

Select the single best option for each question stem

1 Family studies have shed light on psychopathology in offspring of parents with bipolar disorder (familial-risk offspring). Which of the following statements correspond to current findings?

a A positive family history is the strongest predictive factor for development of bipolar disorder

b Approximately $30 \%$ of familial-risk offspring will develop bipolar disorder

c Around $75-80 \%$ of familial-risk offspring with antecedents of major depression will develop bipolar disorder

d Familial-risk offspring are at risk only for bipolar disorders

e All studies confirm the presence of high rates of ADHD in familial-risk offspring.

2 Research methods can influence findings In offspring studies in bipolar disorder we know that:

a cross-sectional studies provide more information about the early trajectory and temporal progression of bipolar disorder than longitudinal studies b longitudinal studies decrease the risk of false-positive diagnoses for bipolar disorder

c all offspring studies have included only well non-proband parents

d researchers diagnosing proband parents and offspring are always masked to family affiliation

e all reported studies have included control groups.

3 As regards clinical findings in offspring studies in bipolar disorder:

a studies have shown lower rates of bipolar, other affective and non-mood psychopathology in familial-risk offspring than in controls

b cross-sectional studies have shown low rates of ADHD

c anxiety symptoms, sleeping problems and increased sensitivity in early childhood precede onset of minor and major mood disorders in familial-risk offspring

d longitudinal studies have shown that the first major affective episode in familial-risk offspring is (hypo)manic in polarity and begins on average in mid to late adolescence

e substance use disorder develops later on in the disorder and is associated with a later age at onset.
4 About $10 \%$ of familial-risk children develop the disorder. Other potential contributing environmental factors for which there is evidence in the literature include:

a advanced maternal age

b low birth weight

c absence of early childhood trauma

d maternal influenza, which may increase the risk of non-psychotic mood episodes

e a low stress load and decreased frequency of life events, which may increase the liability to develop mood disorders.

5 The findings of offspring studies in bipolar disorder support the need for early intervention strategies. Which of the following statements has been explored?

a A clinical staging approach has no relevance to early detection and diagnosis of bipolar disorder

b Psychotherapeutic and family-based interventions could improve outcome

c Early interventions will only benefit offspring at ultra-high risk of developing the illness

d The families, in general, express a negative attitude to genetic testing

e Anti-rumination and sleep regulation strategies have no role to play in early intervention strategies. 